\title{
Review Article \\ The Effect of Periodontitis on Expression of Interleukin-21: A Systematic Review
}

\author{
Archana Mootha, Sankari Malaiappan, N. D. Jayakumar, \\ Sheeja S. Varghese, and Julie Toby Thomas \\ Department of Periodontics, Saveetha Dental College and Hospitals, No. 162, Poonamallee High Road, \\ Velappanchavadi, Chennai 600077, India
}

Correspondence should be addressed to Archana Mootha; archanamootha@gmail.com

Received 21 September 2015; Revised 24 December 2015; Accepted 11 January 2016

Academic Editor: Alexander J. Steven

Copyright ( $) 2016$ Archana Mootha et al. This is an open access article distributed under the Creative Commons Attribution License, which permits unrestricted use, distribution, and reproduction in any medium, provided the original work is properly cited.

\begin{abstract}
Purpose. Inflammation and tissue breakdown are led by an array of inflammatory destructive mediators associated with initiation and progression of inflammatory diseases like periodontitis. Current evidence shows that these inflammatory mediators have a definitive role in the pathogenesis of various systemic diseases with an inflammatory component. Interleukin-21 (IL-21) has been associated with systemic diseases like rheumatoid arthritis and Crohn's disease that follow a chronic inflammatory cascade. Similarly recent studies have associated Interleukin-21 levels with periodontitis. This systematic review was aimed to assess the levels of IL-21 in subjects with periodontitis. Methods. A complete literature search was done in PubMed, Medline, Science Direct, and Cochrane databases and Google Scholar based on the inclusion/exclusion criteria. Six relevant articles were procured. Full text was read individually by two reviewers and data extraction was done based on STROBE statement. Results. After data extraction five observational and one interventional study were obtained. All the studies showed an increased expression of IL-21 in periodontitis and the interventional study showed reduction in IL-21 levels after nonsurgical periodontal therapy (NSP). Conclusion. Interleukin21 levels are higher in periodontitis than controls. With this limited evidence further longitudinal studies are required to consider this as a definitive inflammatory marker.
\end{abstract}

\section{Introduction}

Periodontitis is a chronic inflammatory condition which was initiated by gram-negative organisms present in the tooth supporting structures [1]. Disease progression occurs as a result of host-immune response to bacteria, leading to destruction of connective tissue and alveolar bone [2]. The pathogens present in the subgingival flora produce various endotoxins which are a prerequisite for periodontal disease [3]. These endotoxins in turn activate a host-immune response to the bacterial challenge by stimulating immune cells like polymorphonuclear neutrophils (PMNs), monocytes, B cells, T cells, and fibroblasts [2] to produce various inflammatory mediators such as cytokines $[4,5]$, acute phase proteins [6], and proteolytic enzymes $[7,8]$ that mediate tissue destruction. Progression of the periodontal disease can further trigger the adaptive immune mechanism for release of inflammatory mediators resulting in further periodontal breakdown.

Cytokines are important in expression of the characteristics of the immune response to bacterial endotoxins [9]. Interleukins comprise a large group of cytokines that are naturally occurring glycoproteins produced by the body [10]. They help in recruitment of neutrophils and macrophages to participate and amplify the inflammatory immune reaction [11].

The role of IL-1, IL-6, IL-8, and IL-12 has previously been established in periodontitis [12-15] proving their definitive role in periodontal destruction. Interleukin-21 (IL-21) is an inflammatory cytokine [16] mainly expressed by activated Th1 and Th17 cells which are distinct proinflammatory lineages, and not by Th2 cells in humans pointing out it to be a proinflammatory cytokine [17]. It functions via the receptor IL-21R which is a type I cytokine receptor. Recently 
IL-21 has gained importance as it has been associated with the pathogenesis of inflammatory breakdown in various systemic diseases like rheumatoid arthritis [18], colitis [19], and inflammatory bowel disease [20]. On account of the fact that IL-21 plays a paramount role in inflammation [21], its over production leads to amplification of local inflammation, intensifying tissue damage and destruction [22]. Evidences prove that its regulation in vivo has clinical potential in inflammatory diseases like rheumatoid arthritis [23] and is an area for active research. Since periodontitis and rheumatoid arthritis were previously associated with one another [24] this systematic review was aimed to evaluate the expression of IL21 in periodontitis.

\section{Materials and Methods}

Based on the aim the following structured questions were formulated:

(a) Is there an increase in Interleukin-21 levels in periodontitis?

(b) Are IL-21 levels associated with severity of periodontitis?

(c) Is there a difference in the IL-21 levels between types of periodontitis?

2.1. Search Strategy. The Cochrane, Medline/PubMed, and Science Direct databases and Google Scholar were searched to identify the relevant studies published through June 2015. A detailed search based on MeSH terms and key words was done. A hand search was carried out in the Journal of Periodontology, Journal of Periodontal Research, Journal of Clinical Periodontology, Journal of Periodontal and Implant Science, and International Journal of Periodontics and Restorative Dentistry to check for relevant additional studies. To locate additional studies, the references of the selected articles were hand-searched. No time limits and language restrictions were applied to include all the potentially relevant articles in the review. Selected studies were screened on the basis of the title and abstract. Full text was then procured for the relevant articles which fulfilled the inclusion criteria. The complete search strategy is mentioned in Table 1 and the flowchart is shown in Figure 1.

2.2. Inclusion and Exclusion Criteria. Studies estimating IL-21 levels in serum, saliva, gingival tissue, or GCF of subjects with chronic periodontitis or aggressive periodontitis, regardless of the methodology followed, were included. All animal studies were excluded. Studies estimating IL-21 levels in other oral lesions in subjects without periodontitis were also excluded.

2.3. Screening Methods and Data Extraction. The excluded and included studies are shown in Tables 2 and 3. Each study's methodological quality was assessed based on the STROBE statement by two reviewers (Archana Mootha and Sankari Malaiappan). The studies included in this review were read independently to extract the descriptive and quantitative
TABLE 1: Search methodology.

[[][[][[][[][[][[][[][[][[][[] aggressive periodontitis [MeSH Terms]] OR chronic periodontitis [MeSH Terms]] OR periapical periodontitis [MeSH Terms]] OR gingivitis [MeSH Terms]]] OR periodontitis] OR periodontal disease]]] AND [[[[[IL21] OR interleukin 21] OR IL-21] OR IL21 protein, human] OR Interleukin 21 protein, human]]]*

* Pubmed MESH search.

Potentially relevant articles identified from electronic search, $N=10$

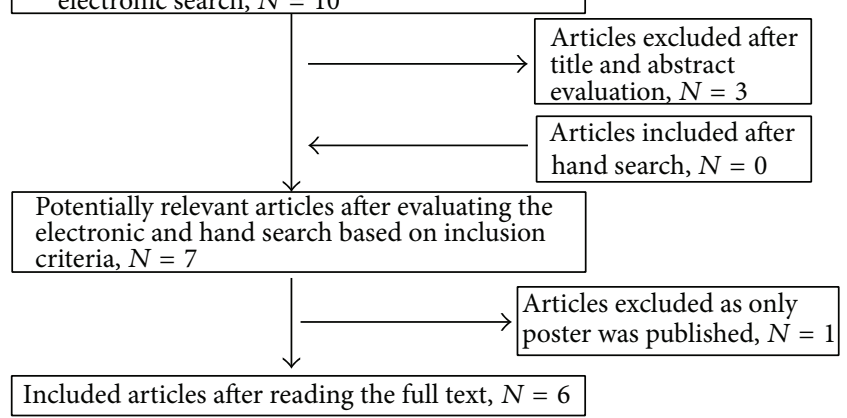

FIGURE 1: Flowchart for selection of articles.

TABLE 2: Excluded studies.

\begin{tabular}{lll}
\hline S. number Citation & Reason for exclusion \\
\hline 1 & Ebersole et al. [4] & $\begin{array}{l}\text { Animal study } \\
\text { IL-21 was estimated in } \\
\text { periapical granuloma in } \\
\text { subjects without } \\
\text { periodontitis }\end{array}$ \\
2 & Araujo-Pires et al. [25] & $\begin{array}{l}\text { Animal study } \\
\text { No abstract/full text } \\
\text { available }\end{array}$ \\
4 & Li et al. [26] & Wang et al. [27]
\end{tabular}

information including citation author, year of publication, study design, study sample, number of participants in control and test groups, method of evaluation of IL-21, levels of IL21, correlation of IL-21 levels with clinical parameters, and results.

\section{Results}

The electronic databases and hand search yielded a total of ten articles (Figure 1). Four articles [4, 25-27] were excluded and the reasons for exclusion are given in Table 2. Full texts for six articles were then procured and data extraction was done. A description of each study is given in Table 3.

The final six studies included five cross-sectional [28, 29, 31-33] and one interventional study [30]. All cross-sectional studies showed increased expression of IL-21 in periodontitis.

The first study estimated salivary IL-21 using ELISA and reported that IL-21 was expressed in all the groups (mild/moderate/no OSAS) with a significant positive correlation with CAL $(p=0.02)$ [28]. 


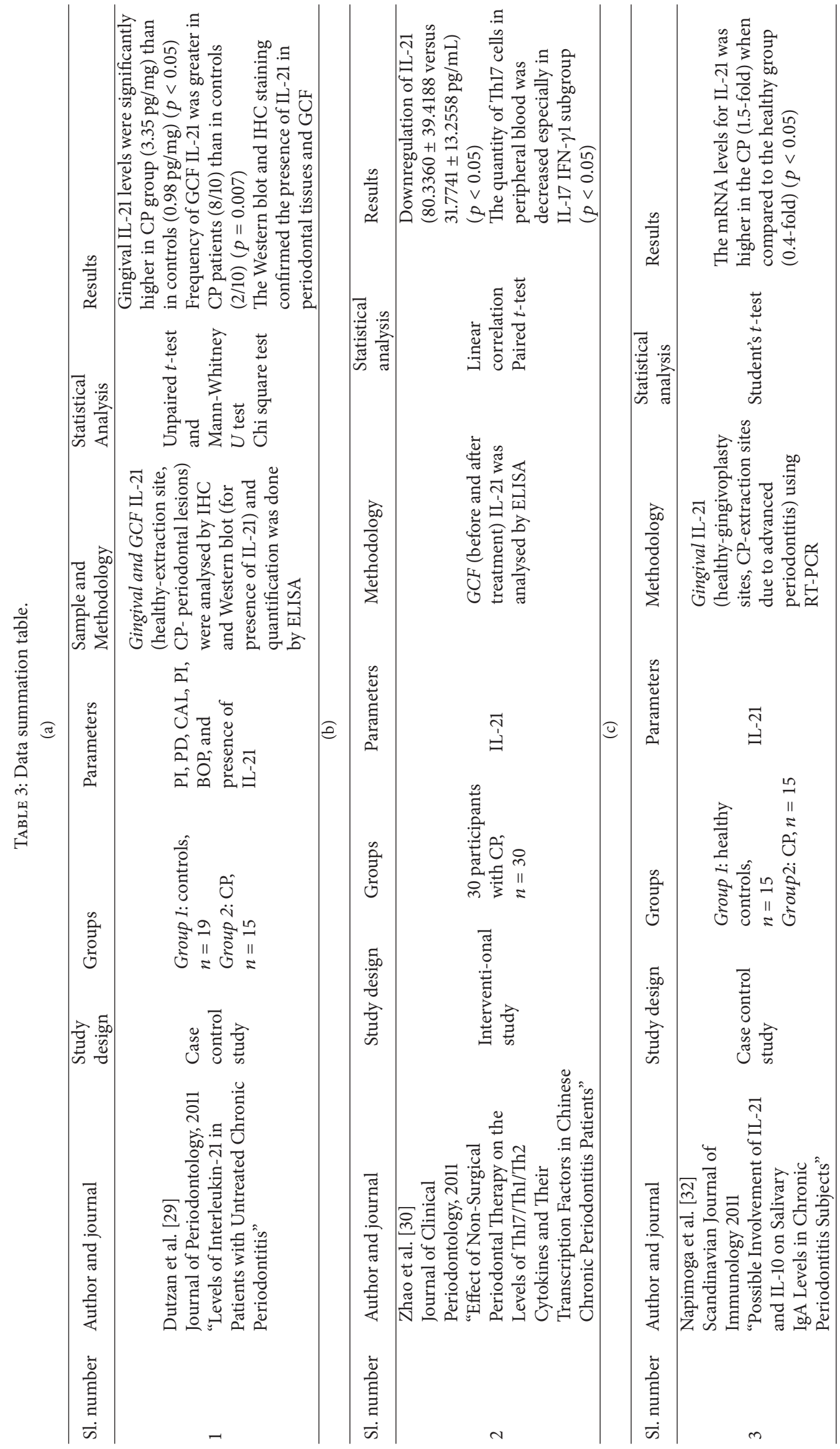




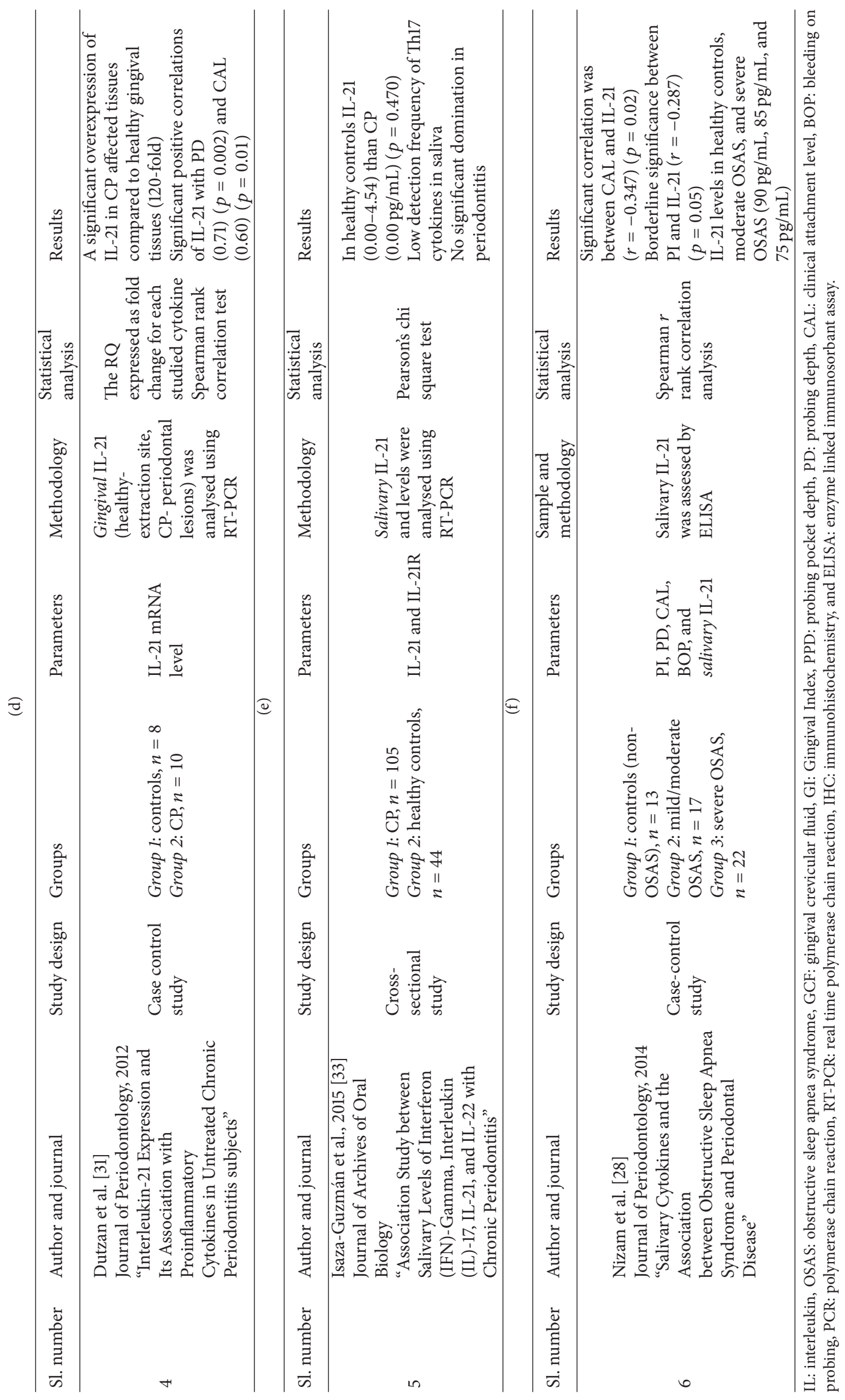


Next study [29] performed qualitative analysis of gingival tissue samples IL-21 using immunohistochemistry and quantitative analysis of salivary and gingival tissue IL-21 using western blot. Gingival tissue IL-21 $(3.35 \mathrm{pg} / \mathrm{mL})$ and GCF IL$21(8 / 10)$ were higher in CP (chronic periodontitis) compared to controls.

Another study showed increased expression of IL-21 in gingival tissue samples using PCR, which were 1.5 times more in CP than controls (0.3) [32]. Another study [31] compared IL-21 levels in gingival tissue between $\mathrm{CP}$ and healthy controls using RT-PCR. CP group showed 120-fold increase of IL-21 level and showed a direct positive correlation with probing depth $(p=0.02)$ and CAL $(p=0.02)$.

One study showed reduced expression of salivary IL-21 in periodontitis using ELISA [33]. Salivary estimation of IL21 using ELISA in CP (smokers were included) showed no significant domination of this cytokine $(0.00-4.5 \mathrm{pg} / \mathrm{mL})$ and it was concluded that the detection of IL-21 has no predictive value in health/disease.

In the interventional study, the GCF IL-21 levels were increased in CP group and there was a reduction in IL-21 levels after nonsurgical periodontal therapy when estimated using ELISA [30].

Due to variations in the study population, sample source and the methodology of estimation of IL-21 the data obtained were heterogeneous. One study was conducted in Brazilian [32], one study in Turkish [28], and one study in Chinese population [30], whereas three studies were conducted in the American [29, 31, 33] population. Varied sources were used for analysis of IL-21. Two studies used GCF [29, 30], two studies used gingival tissue $[29,31,32]$, and two studies used saliva $[28,33]$. All the samples showed increased IL-21 levels in periodontitis, among which GCF [30] showed maximum detection of IL-21 $(80.336 \mathrm{pg} / \mathrm{mL})$. Several methodologies were used for qualitative and quantitative analysis of IL-21. Three studies detected IL-21 using RT-PCR [31, 32], one study used western blot and immunohistochemistry [29], and three studies used ELISA [28-30, 33] for analysis of IL-21. All methodologies showed increased IL-21 levels in periodontitis, among which ELISA showed maximum detection of IL-21 [30]. Due to the heterogeneity of the data it was not feasible to pool the data to perform a meta-analysis. Only two studies showed significant correlation of IL-21 level with periodontal disease severity [31,32]. They found a significant correlation of IL-21 with clinical attachment loss (CAL) and borderline significance between plaque index and IL-21. As CAL is the most reliable indicator of periodontal tissue destruction, this correlation indicates better that IL-21 levels increase with the severity of periodontitis.

\section{Discussion}

Periodontitis is a chronic inflammatory disease that affects the supporting structures of the teeth and is considered as one of the most common reasons for tooth loss [34]. It is one of the most common pathologies of bone and an important modifying factor of several chronic inflammatory systemic diseases like cardiovascular disease and obesity [35]. Evidence shows association of IL-21 with various systemic conditions such as coronary artery disease [36], rheumatoid arthritis [18], and inflammatory bowel disease [19]. Much about IL-21 has been studied in rheumatoid arthritis (RA), and researchers have reported elevated serum and synovial tissues levels of this cytokine in RA as the disease severity increased [37].

Periodontitis is a chronic inflammatory condition which shows increased local and systemic levels of inflammatory mediators and markers of inflammation like IL-21, and a direct link has been established between periodontitis and rheumatoid arthritis. In this regard, this systematic review aimed to evaluate the expression of IL-21 in periodontitis.

Clinically, periodontal disease is characterized by increase in probing depth and a decrease in clinical attachment level. The ultimate determinant of disease progression and clinical outcome is the immune response of host, which involves the generation of cytokines, activation of osteoclasts, and the recruitment of inflammatory cells like lymphocytes, PMNs, and antigen presenting cells to the site [2]. The recruitment and activation of these host cells lead to overproduction of proinflammatory cytokines like IL-1, IL-6, IL12 , and IL-21 along with other host-destructive mediators that enhance host destruction and activate resident cells to produce proteases which further amplify the host mediated periodontal destruction.

A limited number of eligible studies were identified in this systematic review including five observational [23, 24, $28,29,31-33$ ] and one interventional studies [30]. The results of this systematic review show that five $[23,24,28,29,31-$ 33] out of six studies show an increase in IL-21 levels in chronic periodontitis subjects and one study [33] showed no increase in periodontitis. The interventional study [30] showed a reduction in IL-21 levels following nonsurgical therapy.

Nizam et al. [28] hypothesized that OSAS may predispose patients to periodontal disease and associated the salivary cytokines with OSAS severity. He found that salivary IL6 and IL-33 were similar in OSAS groups but significantly higher than control groups, whereas IL-1 $\beta$, IL-21, and PTX were similar in all groups with strong and weak positive correlation of IL-21 with CAL and PI, respectively. The authors concluded, it is likely that elevated IL-6 reflects the degree of subclinical inflammation in periodontal tissues, which can be a link between periodontal disease and OSAS, and the presence or severity of OSAS does not affect the level of IL-1 $\beta$ and IL-21 in either plasma or saliva.

Dutzan et al. [31] showed an increase in IL-21 levels in chronic periodontitis, a positive correlation of IL-21 with PD, CAL, IL-1 $\beta$, IL-6, and Th17 cytokines like IL-17 and IL-23, and a negative correlation with anti-inflammatory cytokines like IL-10 and TGF- $\beta 1$ [31].

IL-21 influences the actions of various immune cells like Natural Killer (NK) cells, PMNs, and macrophages. NK cells are a part of the innate immune response and serve as a first line of defence against invading pathogens. The development and activation of NK cells are intrinsically dependent on the activity of class I cytokine receptors, and cells treated with IL-21 displayed enhanced effector functions with greater cytolytic activity. IL-21 increased PMN migration to the local 
inflammatory site and caused greater PMN mediated tissue injury. IL-21 also complemented the phagocytic ability of macrophages. In addition to its action in the innate immune response, it also parades the action of the adaptive immune response. IL-21 drives terminal differentiation of B cells into plasma cells, favours the effector functions of T cell, and accelerates memory cell formation for rapid action on encounter of a pathogen for the second time. Furthermore, IL- 6 and IL-21 can stimulate Th17 cells itself to produce increased IL21. Altogether, IL-21 exaggerates the host-immune response and intensifies the local inflammatory actions, and these data support an important role of IL-21 in the pathogenesis of periodontal disease.

Salivary levels of IgA were increased in periodontitis subjects along with gingival levels of IL-21. As it was previously discussed, IL-21 not only controls Th17 activity, but also controls B cell proliferation and maturation into plasma cells; the elevated IgA levels in periodontitis may be explained, as $\operatorname{IgA}$ is predominantly produced by $\mathrm{B}$ cells and both IgA and IL-21 are increased in periodontitis compared to healthy controls. IgA is the most predominant Ig secreted by oral mucosal sites and is considered as the most important protein against microbial defence. This indicated the role of IL-21 in Ig-isotype switching in chronic periodontitis and its influence on the adaptive immune response and the immunomodulation of oral mucosa under the challenge of periodontal pathogens [32]. In contrast to these findings, Isaza et al. detected low frequency of Th17 cytokines in saliva samples and concluded that its determination is useless for the detection of disease presence and/or its severity.

After nonsurgical periodontal therapy, Zhao et al. found that Th17 cells might participate in the development of periodontitis by upregulating the expression of cytokines IL17 and IL-21; meanwhile, Th1 cells increased the expression of IFN- $\gamma$, but Th2 cells inhibited the expression of IL- 4 . He concluded that nonsurgical treatment might decrease the expression of Th17-related cytokines, IL-17 and IL-21, and Th1-related cytokine IFN-g, while increasing the expression of Th2 related cytokine IL-4, thus contributing to the relief of periodontal inflammation [30].

While all the cross-sectional studies showed elevated IL-21 levels in periodontitis, one recent study [33] showed contradictory results by reporting no significant domination of Th17 cytokines in chronic periodontitis. An interesting finding is that there is no positive correlation of smokers with IL-21 levels. No scientific rationale explaining the results is mentioned in the article.

In addition, studies conducted in nonperiodontal oral lesions and animal studies also showed an increase in IL-21 levels in disease state compared to controls. On estimation of IL-21 levels in periapical granulomas, it was demonstrated that IL-21 along with IL-17A, TNF- $\alpha$ (tumour necrosis factor$\alpha$ ), and IFN- $\gamma$ were higher in active periapical granulomas, while IL-4, IL-9, IL-10, and IL-22 were higher in inactive periapical granulomas. In the secondary lymphoid organs CD4+ $\mathrm{T}$ cell subset found in the $\mathrm{B}$ cell follicles was described as a major contributor to B cell-mediated antibody responses and an important source of IL-21. Since B cells are predominant in chronic lesions like periapical granulomas, it would be possible to speculate that IL-21 contributes to a Tfh-B cell response axis in periapical areas, similar to that of tertiary lymphoid tissues associated with chronic infection sites. In view of B cells being potential sources of RANKL, Tfh-B cell axis can directly drive the activity of the lesion via RANKL production [34].

Animal study conducted in rhesus monkeys by Ebersole et al. [4] reported that disease initiation/progression was characterized by overexpression of Th17/Treg cytokine genes (IL-1 $\beta$, IL-6, TGF $\beta$, and IL-21) and downregulation of Th1/Th2 cytokine genes (IL-18 and IL-25). They found that several Th17/Treg cytokine genes positively correlated with tissue destruction genes (TDGs), whereas most Th1/Th2 genes exhibited a negative correlation. Overall their observations were consistent with a cytokine profile driving a Th17 type of response early in the initiation process of periodontitis, followed by a persistence with disease progression.

Isaza-Guzmán et al. [33] showed that rats fed with Actinobacillus actinomycetemcomitans containing mash were compared to control; both B and T cells were increased and activated with enhanced IgG switched Ig in 2 weeks. Bone resorption was evident at 12 weeks with early increase in the levels of IL-21 along with IL-2, macrophage-inhibiting factor, IL-19, TNF- $\alpha$, and CD40 ligand, CD70, while IL-16, TNF- $\alpha$, lymphotoxin- $\beta$, IFN- $\alpha$ l, and IL-1RN were increased from T cells indicating that adaptive immunity appears crucial for bone resorption.

The results of this review conclude that (1) IL-21 was elevated in subjects with chronic periodontitis compared to controls, (2) their levels were correlated with clinical severity of periodontitis (only in 2 studies, and values were given only in one study), and (3) nonsurgical periodontal therapy downregulated IL-21 levels. There are no studies based on comparison of IL-21 levels among types of periodontitis. The present review considers only published data for the interpretation of results and the raw data was not procured form the authors of unpublished studies.

This systematic review included cross-sectional studies (level III) [38] and thus indicates a low level of evidence to prove the definitive role of IL-21 in periodontitis. Future longitudinal studies are required to prove the definitive role of IL-21 in periodontitis and compare IL-21 levels among types of periodontitis. Further research in the field of Interleukin21 could throw light on a better understanding of its role in the pathogenesis of tissue destruction in periodontitis.

\section{Conflict of Interests}

The authors declare that there is no conflict of interests regarding the publication of this paper.

\section{References}

[1] H. Loe, "The role of bacteria in periodontal diseases," Bulletin of the World Health Organization, vol. 59, no. 6, pp. 821-825, 1981.

[2] G. J. Seymour, "Importance of the host response in the periodontium," Journal of Clinical Periodontology, vol. 18, no. 6, pp. 421-426, 1991. 
[3] L. Kesic, J. Milasin, M. Igic, and R. Obradovic, "Microbial etiology of periodontal disease," Facta Universitatis Series: Medicine and Biology, vol. 15, no. 1, pp. 1-6, 2008.

[4] J. L. Ebersole, S. Kirakodu, M. J. Novak et al., "Cytokine gene expression profiles during initiation, progression and resolution of periodontitis," Journal of Clinical Periodontology, vol. 41, no. 9, pp. 853-861, 2014.

[5] D. T. Graves, "The potential role of chemokines and inflammatory cytokines in periodontal disease progression," Clinical Infectious Diseases, vol. 28, no. 3, pp. 482-490, 1999.

[6] R. C. Page, "The role of inflammatory mediators in the pathogenesis of periodontal disease," Journal of Periodontal Research, vol. 26, no. 3, pp. 230-242, 1991.

[7] F. Trindade, F. G. Oppenheim, E. J. Helmerhorst, F. Amado, P. S. Gomes, and R. Vitorino, "Uncovering the molecular networks in periodontitis," Proteomics-Clinical Applications, vol. 8, no. 9-10, pp. 748-761, 2014.

[8] H. M. Fullmer, "Collagenase and periodontal diseases: a review," Journal of Dental Research, vol. 50, no. 2, pp. 288-291, 1971.

[9] S. Stenger and M. Röllinghoff, "Role of cytokines in the innate immune response to intracellular pathogens," Annals of the Rheumatic Diseases, vol. 60, no. 3, pp. iii43-iii46, 2001.

[10] R. E. Elmslie, S. W. Dow, and G. K. Ogilvie, "Interleukins: biological properties and therapeutic potential," Journal of Veterinary Internal Medicine, vol. 5, no. 5, pp. 283-293, 1991.

[11] M. Akdis, S. Burgler, R. Crameri et al., "Interleukins, from 1 to 37 , and interferon- $\gamma$ : receptors, functions, and roles in diseases," The Journal of Allergy and Clinical Immunology, vol. 127, no. 3, pp. 701.e70-721.e70, 2011.

[12] S. P. Engebretson, J. T. Grbic, R. Singer, and I. B. Lamster, "GCF IL-1 $\beta$ profiles in periodontal disease," Journal of Clinical Periodontology, vol. 29, no. 1, pp. 48-53, 2002.

[13] C. R. Irwin and T. T. Myrillas, "The role of IL-6 in the pathogenesis of periodontal disease," Oral Diseases, vol. 4, no. 1, pp. 43-47, 1998.

[14] A. Harada, N. Sekido, T. Akahoshi, T. Wada, N. Mukaida, and K. Matsushima, "Essential involvement of interleukin-8 [IL-8] in acute inflammation," Journal of Leukocyte Biology, vol. 56, pp. 559-564, 1994.

[15] I.-S. Tsai, C.-C. Tsai, Y.-P. Ho, K.-Y. Ho, Y.-M. Wu, and C.C. Hung, "Interleukin-12 and interleukin-16 in periodontal disease," Cytokine, vol. 31, no. 1, pp. 34-40, 2005.

[16] C. de Rham, S. Ferrari-Lacraz, S. Jendly, G. Schneiter, J.-M. Dayer, and J. Villard, "The proinflammatory cytokines IL-2, IL15 and IL-21 modulate the repertoire of mature human natural killer cell receptors," Arthritis Research and Therapy, vol. 9, no. 6, article R125, 15 pages, 2007.

[17] M. Sarra, F. Pallone, and G. Monteleone, "Interleukin-21 in chronic inflammatory diseases," BioFactors, vol. 39, no. 4, pp. 368-373, 2013.

[18] T. K. Rasmussen, T. Andersen, M. Hvid et al., "Increased interleukin 21 (IL-21) and IL-23 are associated with increased disease activity and with radiographic status in patients with early rheumatoid arthritis," The Journal of Rheumatology, vol. 37, no. 10, pp. 2014-2020, 2010.

[19] K. Gerlach, C. Daniel, H. A. Lehr et al., "Transcription factor NFATc2 controls the emergence of colon cancer associated with IL-6-dependent colitis," Cancer Research, vol. 72, no. 17, pp. 4340-4350, 2012.

[20] C. Abraham and J. Cho, "Interleukin-23/Th17 pathways and inflammatory bowel disease," Inflammatory Bowel Diseases, vol. 15, no. 7, pp. 1090-1100, 2009.
[21] R. Nurieva, X. O. Yang, G. Martinez et al., "Essential autocrine regulation by IL-21 in the generation of inflammatory T cells," Nature, vol. 448, no. 7152, pp. 480-483, 2007.

[22] T. Onoda, M. Rahman, H. Nara et al., "Human $\mathrm{CD} 4^{+}$central and effector memory T cells produce IL-21: effect on cytokinedriven proliferation of $\mathrm{CD}^{+} \mathrm{T}$ cell subsets," International Immunology, vol. 19, no. 10, pp. 1191-1199, 2007.

[23] F.-L. Yuan, W. Hu, W.-G. Lu et al., "Targeting interleukin-21 in rheumatoid arthritis," Molecular Biology Reports, vol. 38, no. 3, pp. 1717-1721, 2011.

[24] F. B. Mercado, R. I. Marshall, A. C. Klestov, and P. M. Bartold, "Relationship between rheumatoid arthritis and periodontitis," Journal of Periodontology, vol. 15, pp. 1090-1100, 2001.

[25] A. C. Araujo-Pires, C. F. Francisconi, C. C. Biguetti et al., "Simultaneous analysis of $\mathrm{t}$ helper subsets (Th1, Th2, Th9, Th17, Th22, Tfh, Tr1 and Tregs) markers expression in periapical lesions reveals multiple cytokine clusters accountable for lesions activity and inactivity status," Journal of Applied Oral Science, vol. 22, no. 4, pp. 336-346, 2014.

[26] Y. Li, C. Messina, M. Bendaoud, D. H. Fine, H. Schreiner, and V. K. Tsiagbe, "Adaptive immune response in osteoclastic bone resorption induced by orally administered Aggregatibacter actinomycetemcomitans in a rat model of periodontal disease," Molecular Oral Microbiology, vol. 25, no. 4, pp. 275-292, 2010.

[27] Z. M. Wang, J. Zhang, B. S. Pang, L. Q. Zhang, and C. Wang, "Detection of serum interleukin-21 associated with chronic periodontitis," in Proceedings of the 86th General Session of Exhibition of the IADR, Toronto, Canada, July 2008.

[28] N. Nizam, O. K. Basoglu, M. S. Tasbakan, A. Nalbantsoy, and N. Buduneli, "Salivary cytokines and the association between obstructive sleep apnea syndrome and periodontal disease," Journal of Periodontology, vol. 85, no. 7, pp. e251-e258, 2014.

[29] N. Dutzan, C. Rivas, J. García-Sesnich et al., "Levels of interleukin-21 in patients with untreated chronic periodontitis," Journal of Periodontology, vol. 82, no. 10, pp. 1483-1489, 2011.

[30] L. Zhao, Y. Zhou, Y. Xu, Y. Sun, L. Li, and W. Chen, "Effect of non-surgical periodontal therapy on the levels of Th17/Th1/Th2 cytokines and their transcription factors in Chinese chronic periodontitis patients," Journal of Clinical Periodontology, vol. 38, no. 6, pp. 509-516, 2011.

[31] N. Dutzan, R. Vernal, J. P. Vaque et al., "Interleukin-21 expression and its association with proinflammatory cytokines in untreated chronic periodontitis subjects," Journal of Periodontology, vol. 82, pp. 1483-1489, 2012.

[32] M. H. Napimoga, L. H. A. C. Nunes, A. A. B. Maciel et al., "Possible involvement of IL-21 and IL-10 on salivary IgA levels in chronic periodontitis subjects," Scandinavian Journal of Immunology, vol. 74, no. 6, pp. 596-602, 2011.

[33] D. M. Isaza-Guzmán, N. Cardona-Vélez, D. E. Gaviria-Correa, M. C. Martínez-Pabón, M. C. Castaño-Granada, and S. I. Tobón-Arroyave, "Association study between salivary levels of interferon (IFN)-gamma, interleukin (IL)-17, IL-21, and IL-22 with chronic periodontitis," Archives of Oral Biology, vol. 60, no. 1, pp. 91-99, 2015.

[34] H. Löe, A. Anerud, H. Boysen, and M. Smith, "The natural history of periodontal disease in man: the rate of periodontal destruction before 40 years of age," Journal of Periodontology, vol. 49, no. 12, pp. 607-620, 1978.

[35] F. A. Scannapieco and R. J. Genco, "Association of periodontal infections with atherosclerotic and pulmonary diseases," Journal of Periodontal Research, vol. 34, no. 7, pp. 340-345, 1999. 
[36] R. Ding, W. Gao, Z. He et al., "Effect of serum interleukin 21 on the development of coronary artery disease," APMIS, vol. 122, no. 9, pp. 842-847, 2014.

[37] J. Kim, S. Kang, J. Kim, G. Kwon, and S. Koo, "Elevated levels of T helper 17 cells are associated with disease activity in patients with rheumatoid arthritis," Annals of Laboratory Medicine, vol. 33, no. 1, pp. 52-59, 2013.

[38] D. L. Sackett, "Rules of evidence and clinical recommendations on the use of antithrombotic agents," Chest, vol. 95, no. 2, pp. $2-4,1989$. 


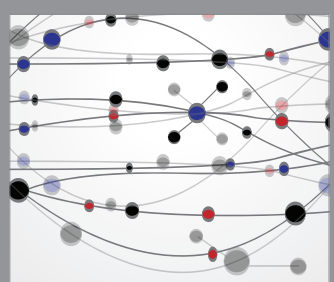

The Scientific World Journal
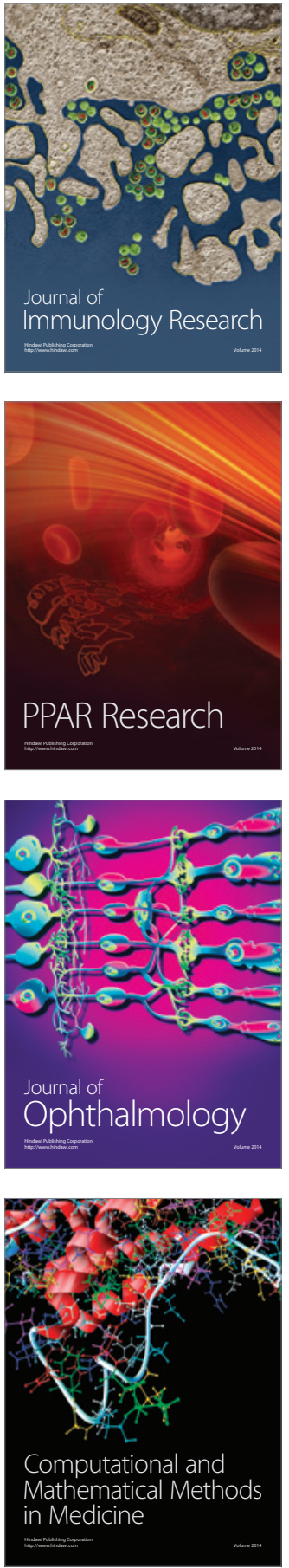

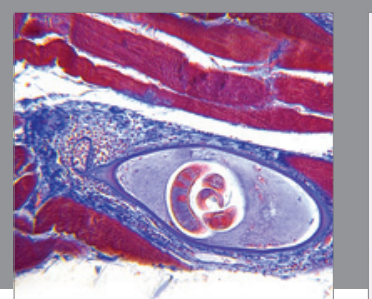

Gastroenterology Research and Practice

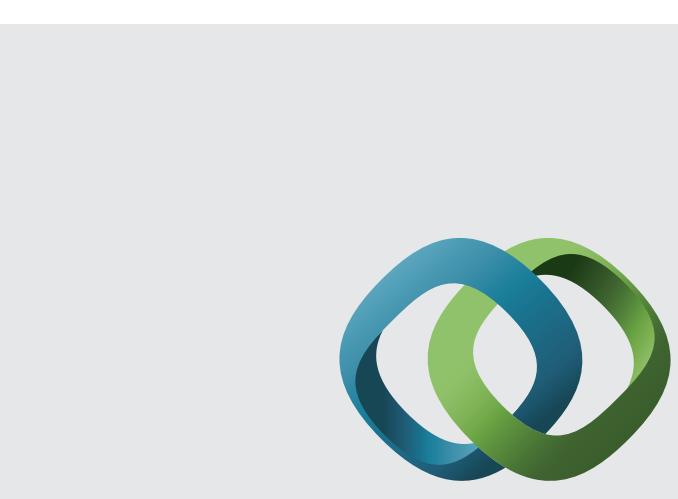

\section{Hindawi}

Submit your manuscripts at

http://www.hindawi.com
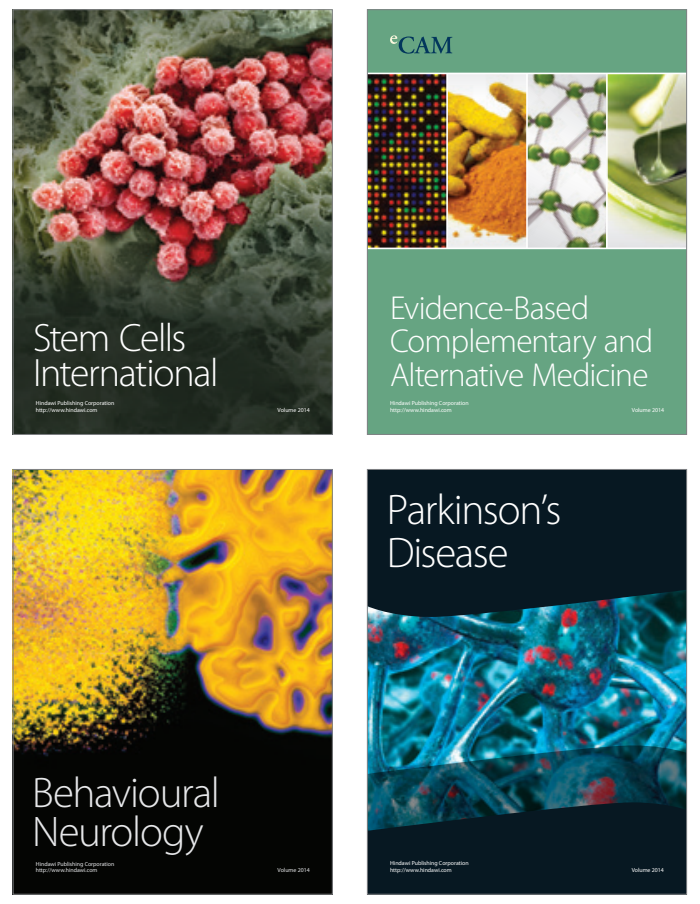
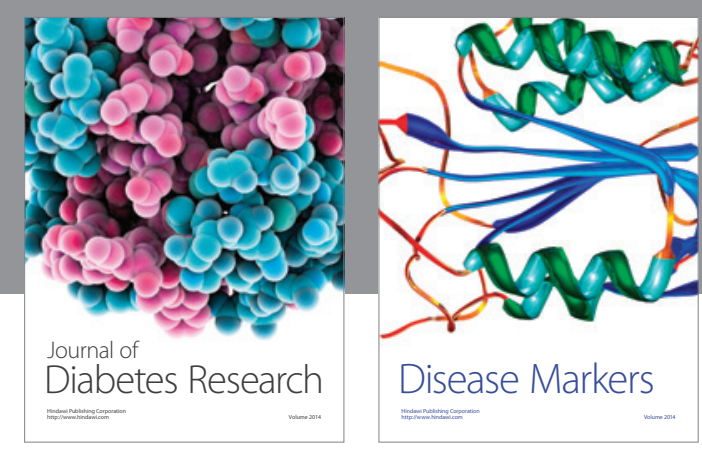

Disease Markers
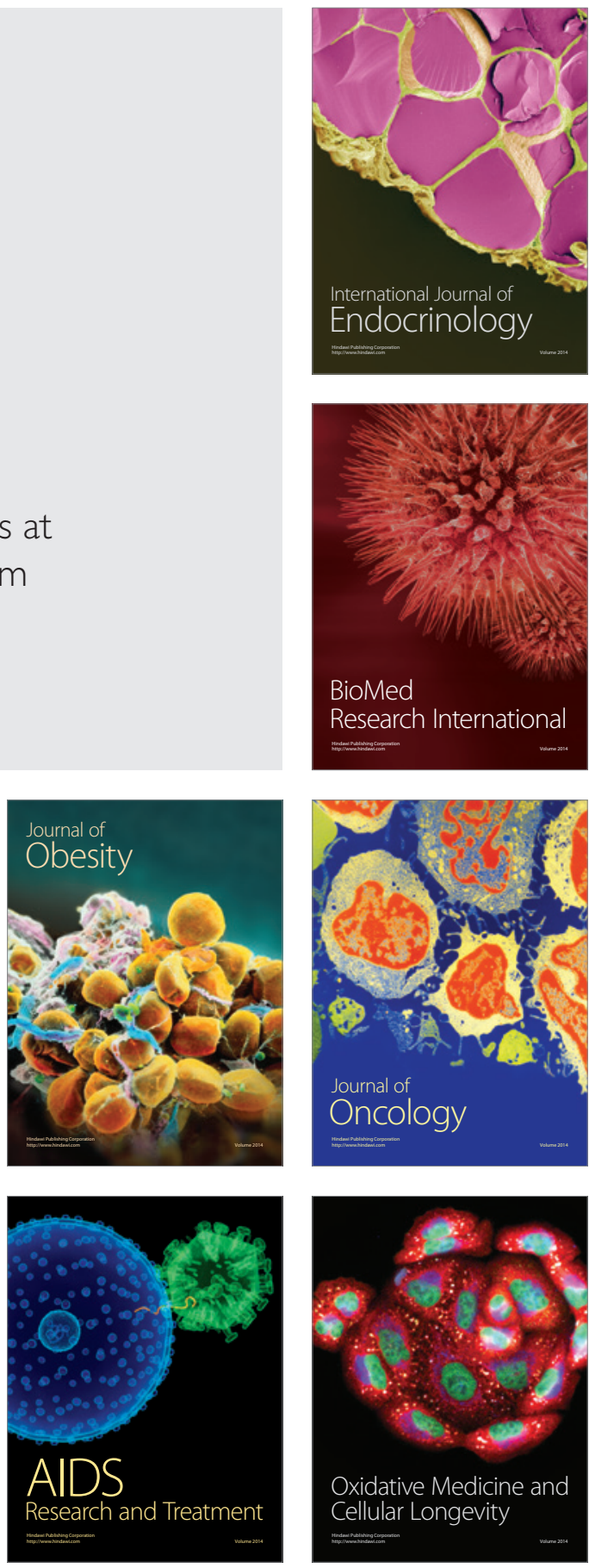